\title{
RELEVANCE OF 18F-FLUORODEOXYGLUCOSE-POSITRON EMISSION TOMOGRAPHY/COMPUTED TOMOGRAPHY IN THE DIAGNOSIS OF ATYPICAL TEMPORAL ARTERITIS
}

\author{
M. Leclercq ${ }^{1}$, L. Goodrich², C. Le Guillou ${ }^{3}$, X. Gbaguidi ${ }^{4}$
}

\begin{abstract}
Atypical form of Giant cell arteritis (GCA), involving the absence of American College of Rheumatology (ACR) criteria, should be suspected before fever or inflammatory syndrome of unknown origin in the elderly. In that case, the diagnosis may be complex insofar as there are many possible infectious, inflammatory or tumorous etiologies. Completion of multiple and / or invasive clinical investigations can be questionable in a frail elderly population. 18F-fluorodeoxyglucose positron emission tomography/computed tomography (FDG-PET/CT) is a non-invasive examination of high sensitivity and specificity in the diagnosis of GCA which may reveal abnormal fixation of the wall of the aorta and its branches. Its negative predictive value close to $90 \%$ is also a strength to exclude the diagnosis. FDG-PET/CT is also effective in the search for differential diagnoses. On the other hand, FDG-PET/CT should not be prescribed in front of typical form of GCA in which ACR criteria are met. This clinical case illustrate the place of FDG-PET / CT in the diagnostic strategy of an inflammatory syndrome in an elderly patient for whom GCA is suspected.
\end{abstract}

Key words: Aged, 18F-fluorodeoxyglucose-positron emission tomography/computed tomography, giant cell arteritis.

\section{Introduction}

A persistent inflammatory syndrome in patients over 60 years old should raise the hypothesis of giantcell arteritis (GCA). Although the classic clinical and biological presentation of the disease is fairly simple to diagnose, it is not so obvious for atypical forms. This clinical case illustrates the place of 18F-fluorodeoxyglucose positron emission tomography / computed tomography (FDG-PET/CT) before fever or chronic inflammatory syndrome with a possible diagnosis of GCA.

\section{Clinical case}

An 88 year old patient was hospitalized for the assessment of an inflammatory syndrome. During the last 4 months had appeared asthenia and fluctuant cognitive impairment. These symptoms altered the activities of daily living. The temperature was $38{ }^{\circ} \mathrm{C}$. The physical examination was normal including present temporal pulse. Biological examinations highlighted an

1. Internal Medicine, Rouen University Hospital, Rouen, France; 2. Geriatrics and Rehabilitation, Bayeux Hospital Center, Bayeux, France; 3. Geriatrics and Internal Medicine, Nîmes University Hospital, Nîmes, France; 4. Geriatrics, Dieppe Hospital Center, Dieppe, France

Corresponding Author: Dr Xavier Gbaguidi, Department of Geriatrics, Dieppe Hospital Center, Avenue Pasteur 76200 Rouen, Email : xgbaguidi@ch-dieppe.fr, Tel : +33 2321472 15, Fax : 0232147492 inflammatory syndrome (CRP: $96 \mathrm{mg} / \mathrm{L}$ ), normocytic anemia $(\mathrm{Hb}: 8 \mathrm{~g} / \mathrm{dL})$ secondary to inflammation, anicteric cholestasis and hypoalbuminemia $(25 \mathrm{~g} / \mathrm{L})$. Infectious causes, especially neuro-meningeal and cardiac infection, were eliminated. Body scanner with injection of iodinated contrast media was performed. Neither tumor anomaly nor inflammatory sign of the aorta or supra aortic trunks were found. A FDG-PET/CT was performed to investigate arterial hypermetabolism in the hypothesis of a GCA. It highlights an arterial inflammation of the superior aortic and iliac trunks in favor of a GCA (figure 1). The systematic temporal artery biopsy (TAB) was positive. Corticosteroid therapy led to the rapid improvement of symptoms.

\section{Discussion}

The GCA is the most frequent vasculitis of the elderly. It usually reaches the branches of the carotid artery but also the large vessels such as the aorta, its branches and the limbs arteries. According to studies, this impairment varies between 17 and $45 \%$ (1) or even up to $83 \%$ (2).

Diagnosis can be complex. The American College of Rheumatology (ACR) criteria (age $>50$ years, recent occurrence of headaches, clinical arterial anomalies, high ESR, histological vasculitis) have good sensitivity and specificity respectively $93 \%$ and $91 \%$ but are not always present. The clinical signs associated with the involvement of the temporal artery may be missing 
as well as the headaches as shown by our patient. In $5 \%$ of cases, only general signs and inflammatory syndrome are present (3), in 15\% it is an isolated fever (2). When negative (15 to $51 \%$ of cases) $(2,4)$, TAB does not eliminate the diagnosis because of the segmental and focal distribution of lesions. Moreover, in the case of inflammatory syndrome or fever of undetermined origin, the oncological, infectious and noninfectious inflammatory etiologies are discussed as much as the hypothesis of GCA. Given this type of clinincal picture associating fever and inflammatory syndrome, the GCA represents approximately $16 \%$ of diagnoses (2). Our patient illustrates this situation perfectly with an aspecific presentation associating asthenia, delirium, fever and inflammatory syndrome.

\section{Figure 1}

Fluorine-18 fluorodeoxyglucose (18F-FDG) positron emission tomography/computed tomography imaging.

Black arrows show pathological FDG uptake in the external carotid (a), subclavian and axillary arteries (b), aorta (c) and iliac arteries (d) that contrast with normal vessel (white arrow)

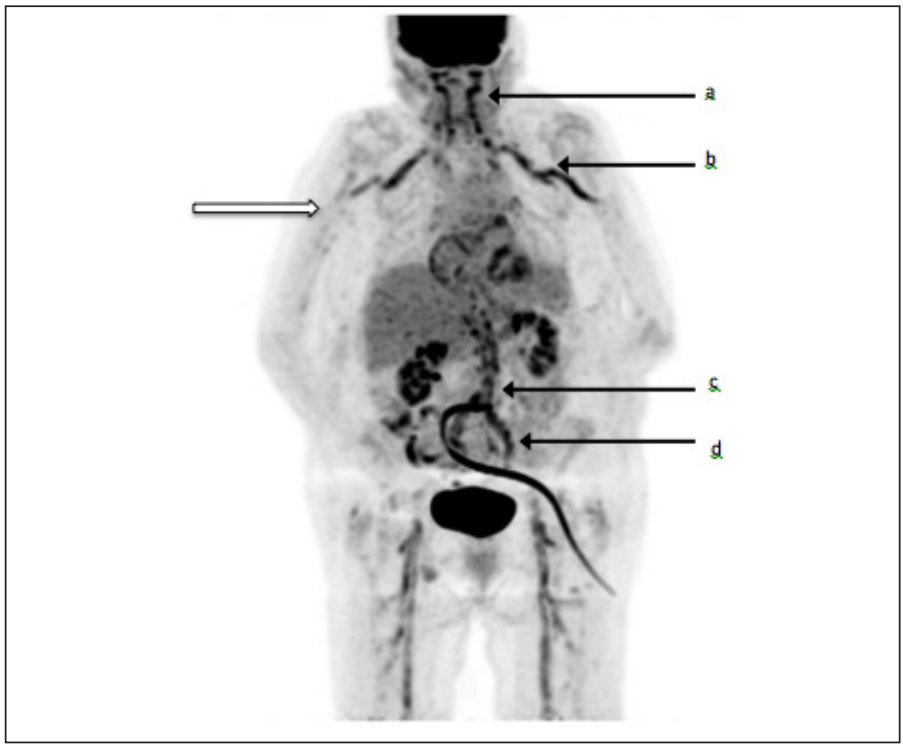

FDG-PET/CT detects tissues with high metabolic activity by injecting a glucose analog bound to a radioactive marker. In the vasculitis, the infiltration of the vascular wall by inflammation cells leads to a fixation on the FDG-PET/CT hence its interest. Two recent meta-analyzes $(5,6)$ show satisfactory performance of in the diagnosis of GCA. Sensitivity and specificity were respectively $80 \%$ to $89.5 \%$ and $89 \%$ to $97.7 \%$. Positive predictive values and negative predictive values (NPV) were respectively $85 \%$ and $88 \%$ (5). False positives may be due to atherosclerosis. A visual fixation of the arterial wall of higher intensity than fixation on liver is a good criterion in favor of vasculitis and not of atheroma (sensitivity of $83 \%$ and specificity of $91 \%$ ) (6). The false negatives correspond to patients with GCA affecting only the temporal arteries without reaching the cervico- thoracic vessels corresponding to the limits of spatial resolution of FDG-PET/CT. However, in this situation, the clinical presentation is usually classical and therefore the ACR criteria are present. Thus, in a recent cohort, all patients with isolated temporal impairment met ACR criteria (7).

These results should motivate the realization of a FDG-PET/CT in front of a persistent inflammatory syndrome or a fever of undetermined origin to look for GCA because once in four it will recover an arterial hypermetabolism compatible with an inflammatory arteritis (8).

With a high NPV, a negative exam would reject the diagnosis of GCA and avoid the use of an invasive procedure. On the contrary, its positivity with typical lesions of intense arterial fixation would be an argument to retain the diagnosis. The FDG-PET/CT is also a useful exam to rule out some differential diagnoses. Indeed, some studies have demonstrated its performance in the etiologic diagnosis of indeterminate origin fever $(9,10)$. On the other hand, when the clinical elements of the ACR criteria exist, the FDG-PET/CT does not find its place in the diagnostic strategy.

Disclosure Statement: No potential conflicts of interest were disclosed; Mathilde Leclercq has nothing to disclose; Laura Goodrich has nothing to disclose; Cédric Le Guillou has nothing to disclose; Xavier Gbaguidi has nothing to disclose.

Ethical standard: All authors have seen and approved the final version of the paper and have agreed to its submission for publication. This clinical case presentation ensure appropriate level of privacy.

\section{References}

1. Marie I, Proux A, Duhaut P et al. Long-term follow-up of aortic involvement in giant cell arteritis: a series of 48 patients. Medicine (Baltimore) 2009; 88: 182-192.

2. Salvarani C, Cantini F, Hunder GG. Polymyalgia rheumatica and giant-cell arteritis. Lancet 2008; 372: 234-245.

3. Gonzales-Gay MA, Garcia-Porrua C, Amor-Dorado JC, Llorca J. Giant cell arteritis without clinically evident vascular involvement in a defined population. Arthritis Rheum 2004; 51: 274-277.

4. Bienvenu B, Ly KH, Lambert M et al. Management of giant cell arteritis: Recommendations of the French Study Group for Large Vessel Vasculitis (GEFA). Rev Med Interne 2016; 37: 154-165.

5. Besson FL, Parienti JJ, Bienvenu B et al. Diagnostic performance of 18F-fluorodeoxyglucose positron emission tomography in giant cell arteritis: a systematic review and meta-analysis. Eur J Nucl Med Mol Imaging 2011; 38: $1764-1772$

6. Soussan M, Nicolas P, Schramm C et al. Management of large-vessel vasculitis with FDG-PET: a systematic literature review and meta-analysis. Medicine (Baltimore) 2015; 94: e622.

7. Czihal M, Zanker S, Rademacher A et al. Sonographic and clinical pattern of extracranial and cranial giant cell arteritis. Scand J Rheumatol 2012; 41: 231 236

8. Lensen K-JDF, Voskuyl AE, van der Laken CJ et al. 18F-fluorodeoxyglucose positron emission tomography in elderly patients with an elevated erythrocyte sedimentation rate of unknown origin. PLoS One 2013; 8: e58917.

9. Meller J, Altenvoerde G, Munzel U et al. Fever of unknown origin: prospective comparison of (18F) FDG imaging with a double-head coincidence camera and gallium-67 citrate SPET. Eur J Nucl Med 2000; 27: $1617-1625$.

10. Dong M, Zhao K, Liu Z, Wang G, Yang S, Zhou G. A meta-analysis of the value of fluorodeoxyglucose-PET/PET-CT in the evaluation of fever of unknown origin. Eur J Radiol 2011; 80: 834-844. 